\title{
Correlation between Endothelin-1 and oxidative stress in apparently healthy obese men
}

\begin{abstract}
Background and objective: Obesity is the risk for many disease conditions and is becoming a global issue. Endothelial dysfunction and oxidative stress are linked to obesity. Endothelin-1 is a useful marker of endothelial dysfunction. Oxidative stress can be assessed by estimation of lipid peroxidation byproduct malondialdehyde. In this study, we tried to find out the correlation between Endothelin-1 and oxidative stress among apparently healthy obese men.
\end{abstract}

Methods: Eighty apparently healthy non-smoker adult men have participated in this study. Their age ranged between 18 and 50 years. Their serum Endothelin-1 (ELISA), malondialdehyde and lipid parameters (biochemical assays) have been estimated.

Results: Subjects were divided into three groups: normal weight ( $n=25)$, overweight $(n=34)$ and obese $(n=26)$ basing on their body mass index. The study shows that Endothelin-1 is significantly correlated with each of Malondialdehyde and body mass index $(r=0.262, P=0.019 ; r=0.65, P<0.001$ respectively). Malondialdehyde is also significantly positively correlated with $\mathrm{BMI}(r=0.378, P=0.001)$.

Conclusion: Endothelial dysfunction and oxidative stress coexist in obesity. But which of them precedes the other? Is still a question needs to be further studied.

Keywords: Obesity; BMI; Endothelin-1; Malondialdehyde.

\section{Introduction}

Overweight and obesity are important risk factors for cardiovascular disease, which is the number one cause of death and accounts for over 17 million deaths every year. The World Health Organization (WHO) estimates that over 1.4 billion people are overweight globally. Once considered a problem only in wealthy countries, WHO estimates show that overweight and obesity are now dramatically on the rise in low and middle-income countries. This is due to a number of factors, including a global shift in diet towards increased energy, fat, salt and sugar intake, and a trend towards decreased physical activity due to the sedentary nature of modern work and transportation, and increasing urbanization. ${ }^{1}$ Atherosclerosis is the most common cause of coronary heart disease.
Endothelial dysfunction initiates atherosclerosis. One of the factors that cause endothelial dysfunction is dyslipidemia (abnormality in any of the lipoprotein fractions), especially elevated low density lipoproteins (LDL) and decreased high-density lipoproteins $(\mathrm{HDL}){ }^{2}$ Endothelin-1 (ET-1), the most potent endogenous vasoactive peptide ever known, 3,4 had been found to be increased in obese subjects with endothelial dysfunction. ${ }^{5}$ On the other hand, oxidative stress, which is a disturbance in the balance between the production of reactive oxygen species or free radicals and antioxidant defenses, may be the unifying mechanism underlying the development of comorbidities in obesity. Evidence suggests that a clustering of sources of oxidative stress exists in obesity: hyperglycemia,

* Department of Medical Physiology, College of Medicine, Hawler Medical University, Erbil, Iraq. 
hyperleptinemia, increased tissue lipid levels, inadequate antioxidant defenses, increased rates of free radical formation, enzymatic sources within the endothelium, and chronic inflammation. ${ }^{6,7}$ Assessment of oxidative stress in the body can be done by estimation of free radicals by various methods. Among the free radicals generated from lipid peroxidation; Malondialdehyde (MDA) is a well-known secondary product has been shown to be a relevant indicator of lipid peroxidation in biological systems. ${ }^{8,9}$ This study aimed to assess whether oxidative stress in the form of malondialdehyde, lipid peroxidation and ET-1 are elevated among a sample of apparently healthy overweight and obese men in Erbil city. This might be of help in the understanding of the converse relation among the parameters mentioned above that help in better understanding of obesity and factors affecting it.

\section{Methods}

Subjects: The study was conducted in Erbil city during July and August 2012. Eighty apparently healthy non-smoker men volunteers participated in this study. The age of them ranged between 18 and 50 years. The volunteers were from Nanakaly hospitals' staff and patients' accompany. The study was designed as an analytical cross-sectional study. Subjects were grouped based on their body mass indices into three groups; normal weight (BMI of $18.5-24.9 \mathrm{~kg} / \mathrm{m}^{2}$ ), overweight (BMI of 2529.9) and obese (BMI 30 and above). Information about their age, history of heart disease and hypertension in first-degree relative(s) were obtained using a questionnaire designed for the study. Height and weight of the participants were measured using a portable stadiometer (SECA Co. -USA) and digital weight scale respectively. BMI was calculated using the formula: Weight $(\mathrm{Kg}) /$ Height. $^{2}$ Waist circumference was measured using a standard tape measure midway between the lower rib and iliac crest. ${ }^{10,11}$ Pulse rate was recorded manually, and blood pressure was measured using a mercury sphygmomanometer after subjects had rested 20-30 minutes in a comfortable position. Inclusion criteria included apparently healthy non-smoker men with no detected signs and symptoms of illnesses by physical examination and on routine medical check up and testing for liver, kidney, heart, brain, blood and metabolic diseases. Exclusion criteria included all other subjects and patients not fulfilling the above mentioned inclusion criteria. This study was a part of MSc thesis and the study proposal was approved by both the scientific and the ethical committee of the College of Medicine, Hawler Medical University, Erbil, Iraq, 2012-2013. A sample of $8 \mathrm{ml}$ of venous blood sample from cubital vein in a sitting position was obtained after 8-12 hours fasting of the subject. ${ }^{12}$ Then, $2 \mathrm{mls}$ of the blood samples were rapidly placed in chilled polypropylene plastic tubes (Stockwell Scientific-USA), centrifuged directly for 20 minutes at $4 C^{0}$ at $2000 x g$ using (Tomy GRX- High Speed Refrigerated Centrifuge, Tokyo, Japan). The serum separated and stored in Protein LoBind PCR clean polypropylene eppendorf tubes (Hamburg-Germany) at $(-25$ to -50$) C^{0}$ and assayed a month later for the estimation of Endothelin-1. The remaining $6 \mathrm{ml}$ of the blood samples were placed in serum separator glass tubes, left aside for 30 minutes to clot and centrifuged for 15 minutes at (3000) r.p.m using Labofuge 200 centrifuge-Germany. The separated serum used for biochemical analysis of (Malondialdehyde, Total Cholesterol, Triglyceride and High Density Lipoproteins). Measurement of serum ET-1 was done using Human Endothelin (1-21) ELISA kit from Creative DiagnosticsNY-USA. The whole procedure was carried out in Nanakaly hospital in Erbil city using Bio-Tek ${ }^{\circledR}$ microplate reader and Bio-Tek ${ }^{\circledR}$ automatic microtiter plate washer-USA. All the steps in the preparation of the samples and standards and the measurement procedures were 
according to the procedure provided by the manufacturer. Lipid peroxidation and lipid profile tests were carried out in the Physiology and Biochemistry research laboratories, College of Medicine, Hawler Medical University, Erbil. Serum MDA was determined by a chemical colorimetric method described by Gutteridge and Halliwell, $1990 .^{13}$ Data of this study were analyzed using the statistical package for the social sciences (version 19.0). All the data are expressed as mean, standard deviation (SD) and/or standard error (SE). Statistical analysis was used according to the Student's independent t-test for comparing means and ANOVA. Pearson's correlation coefficient was also used to correlate the measured parameters. A $P$ value of 0.05 or less was considered to be statistically significant.

\section{Results}

The study groups as per their weight are listed in Table 1. The mean serum ET-1 of the mentioned groups is compared using ANOVA. The difference among study groups was statistically highly significant (Table 2).

Table 1: Anthropometric measures of the normal weight, overweight and obese groups of the study.

\begin{tabular}{lcccc}
\hline $\begin{array}{l}\text { Anthropometric measures } \\
\text { as Mean } \pm \text { SD }\end{array}$ & $\begin{array}{c}\text { Normal Weight } \\
\mathbf{N = 2 5}\end{array}$ & $\begin{array}{c}\text { Overweight } \\
\mathbf{N = 3 4}\end{array}$ & $\begin{array}{c}\text { Obese } \\
\mathbf{N = 2 1}\end{array}$ & $\boldsymbol{P}$ value \\
\hline Age(years) & $30.12 \pm 8.35$ & $32.91 \pm 7.11$ & $35.19 \pm 8.77$ & 0.102 \\
Weight(kgs) & $66.06 \pm 6.77$ & $79.76 \pm 6.61$ & $97.95 \pm 10.63$ & $<0.001$ \\
Height(m) & $1.72 \pm 0.07$ & $1.70 \pm 0.06$ & $1.72 \pm 0.07$ & 0.411 \\
BMl(kg/m $\left.{ }^{2}\right)$ & $22.23 \pm 1.61$ & $27.45 \pm 1.41$ & $32.94 \pm 2.8$ & $<0.001$ \\
$\%$ Body Fat & $17.4 \pm 3.38$ & $24.31 \pm 2.4$ & $31.42 \pm 3.15$ & $<0.001$ \\
Waist circumference $(\mathrm{cm})$ & $80.84 \pm 7.23$ & $93.15 \pm 7.9$ & $105.57 \pm 7.88$ & $<0.001$ \\
\hline
\end{tabular}

Table 2: Serum endothelin-1 values of the normal weight, overweight and obese groups, their comparison and statistical evaluation.

\begin{tabular}{lcc}
\hline Study groups & $\begin{array}{c}\text { Serum ET-1 (fmol/ml) } \\
\text { Mean } \pm \text { SE }\end{array}$ & $P$ value \\
\hline Normal weight $(n=25)$ & $0.966 \pm 0.07$ & $<0.05$ \\
Overweight $(n=34)$ & $1.367 \pm 0.12$ & \\
Normal weight $(n=25)$ & $0.966 \pm 0.07$ & $<0.001$ \\
Obese $(n=21)$ & $2.534 \pm 0.22$ & \\
Overweight $(n=34)$ & $1.367 \pm 0.12$ & $<0.001$ \\
Obese $(n=21)$ & $2.534 \pm 0.22$ & \\
\hline
\end{tabular}


There was a significant moderate positive correlation between ET-1 and BMI ( $r=0.65, P<0.001)$ as shown in Figure 1. Mean serum Malondialdehyde of the study

Table 3: Mean values of serum malondialdehyde of the study groups, their comparison, and statistical evaluations.

\begin{tabular}{lcc}
\hline Study groups & $\begin{array}{c}\text { Serum MDA }(\mu \mathrm{mol} / \mathrm{L}) \\
\text { Mean } \pm \text { SE }\end{array}$ & $\boldsymbol{P}$ value \\
\hline Normal weight $(\mathrm{n}=25)$ & $1.16 \pm 0.05$ & $<0.001$ \\
Overweight $(\mathrm{n}=34)$ & $1.73 \pm 0.08$ & \\
Normal weight $(\mathrm{n}=25)$ & $1.16 \pm 0.05$ & $<0.001$ \\
Obese $(\mathrm{n}=21)$ & $2.3 \pm 0.36$ & $>0.05$ \\
Overweight $(\mathrm{n}=34)$ & $1.73 \pm 0.08$ & \\
Obese $(\mathrm{n}=21)$ & $2.3 \pm 0.36$ & \\
\hline
\end{tabular}

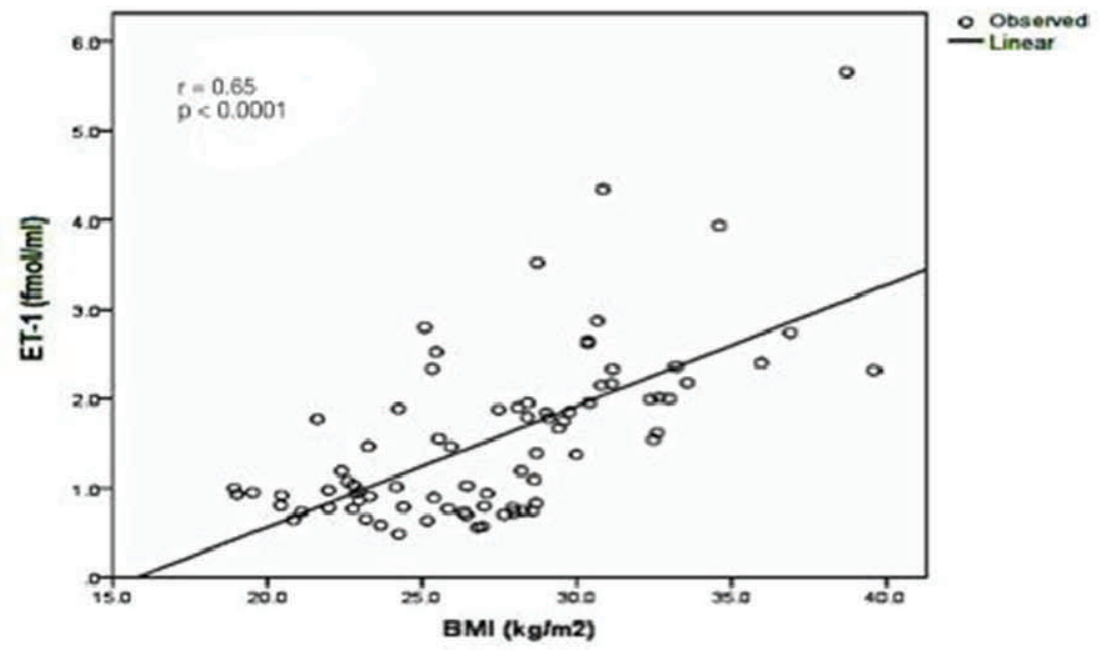

Figure 1: Correlation between Endothelin -1 and body mass index.

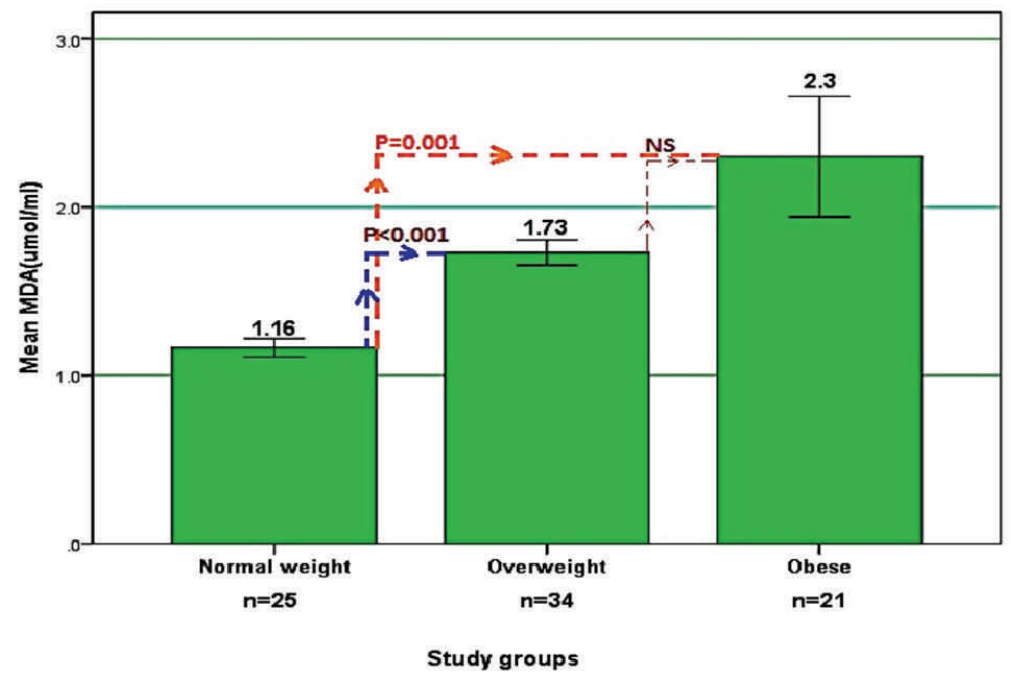

Figure 2: Mean serum malondialdehyde of the study groups. 
Serum MDA is weakly correlated with BMI $(r=0.378)$ and that correlation is statistically significant $(P=0.001)$ as shown in Table 4. Although serum ET-1 of the study subjects was weakly correlated with MDA but turned out to be statistically significant $(r=0.262, P=0.019)$ as shown in Figure 3.

\section{Discussion}

Estimating the level of ET-1 is of crucial importance as one of the best markers of endothelial dysfunction. ${ }^{14}$ In the present study, the overweight and obese subjects showed levels of ET-1 that are higher than in normal weight group, with statistically significant difference. The positive correlation between ET-1 and BMI that has been described by Maeda et al. ${ }^{15}$ and Lilyasari et al., ${ }^{16}$ was comparable to that of the present study. Even more,
Maeda et al. $^{15}$ have found that weight reduction in obese men significantly decreased plasma ET-1 concentration supporting the opinion that obesity in man is associated with endothelial dysfunction. Accumulation of adipose tissue in overweight and obesity creates an inflammatory response with activation of macrophages and release of inflammatory mediators that, in turn, causes insulin resistance and dyslipidemia, a condition that proceeds to atherosclerosis and thrombin formation causing vascular resistance and tissue hypoxia with raised blood pressure. A consequence of that is the increased mechanical and shear stress on the endothelium lining the blood vessels. All conditions mentioned above are known stimulators of endothelial dysfunction and release of ET-1.,17 The present study has viewed that obesity is

Table 4: Correlation among BMI, ET-1, and MDA.

\begin{tabular}{lcc}
\hline Correlations & r values & $\boldsymbol{P}$ value \\
\hline BMI vs. ET-1 & 0.65 & $<0.001$ \\
BMI vs. MDA & 0.38 & $<0.001$ \\
ET-1 vs. MDA & 0.322 & $<0.005$ \\
\hline
\end{tabular}

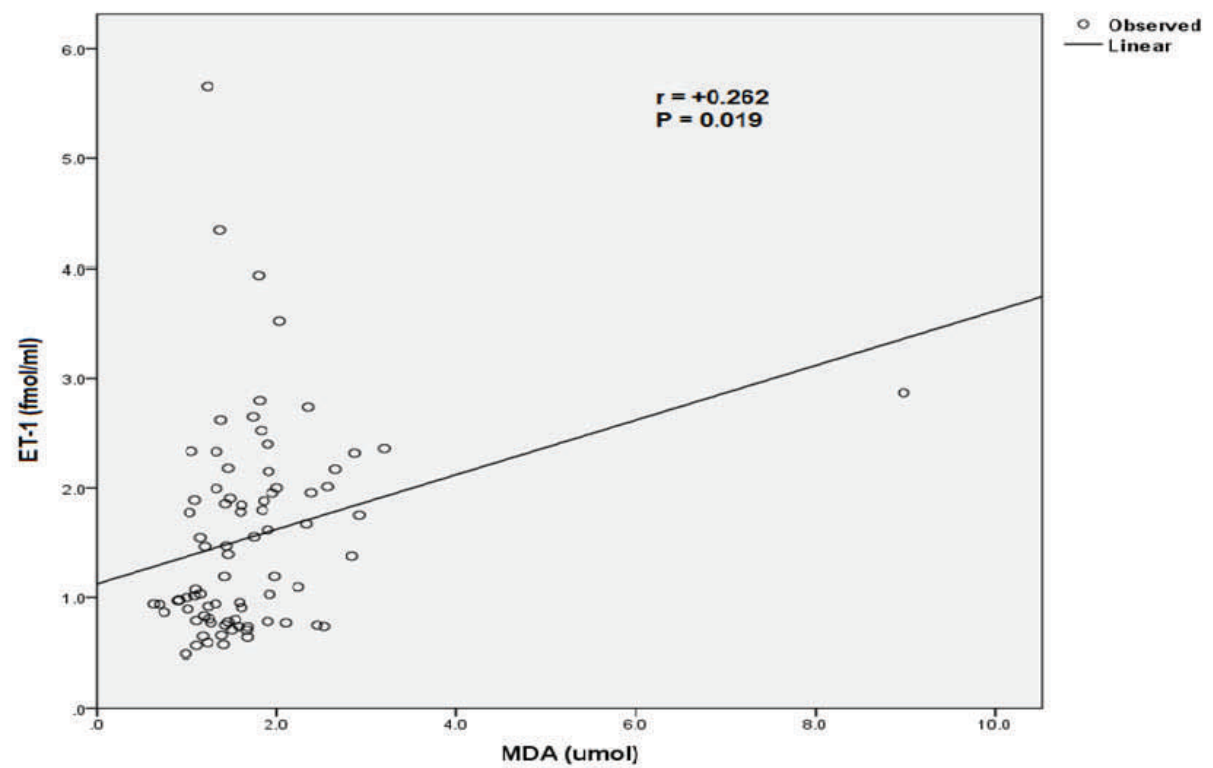

Figure 3: Correlation between ET-1 and MDA. 
associated with oxidative stress in the form of positive correlation between MDA and BMI that is statistically highly significant. These results were comparable to those obtained by Selvakumar et al. ${ }^{18}$ The positive association between obesity and oxidative stress can be explained as; obesity increases the mechanical and metabolic load on the myocardium, thus increasing myocardial oxygen consumption. A negative consequence of the elevated myocardial oxygen consumption is the production of reactive oxygen species (ROS) such as superoxide, hydroxyl radical and hydrogen peroxides from the increased mitochondrial respiration. ${ }^{6}$ Another mechanism by which obesity can independently cause increased lipid peroxidation is progressive and cumulative cell injury resulting from pressure from the large body mass. Cell injury causes the release of cytokines, especially tumor necrosis factor alpha (TNF- $\alpha$ ) which generates ROS from the tissues which in turn cause lipid peroxidation. ${ }^{19}$ The positive correlation between ET-1 and MDA in the present study's subjects was comparable to that described by other researchers, indicating that oxidative stress and endothelial dysfunction coexist. ${ }^{17,20}$

\section{Conclusion}

Endothelial dysfunction exists in obesity and ET-1 can be a useful marker of it. It is also clear that endothelial dysfunction, oxidative stress, and dyslipidemia coexist in obesity, and there is a weak correlation among ET-1, BMI and MDA.

\section{Conflicts of interest}

The authors report no conflicts of interest.

\section{References}

1. Obesity and overweight. World Health Organization (WHO); 2012. Fact Sheet No. 311. (Accessed February 7, 2014), available from :http://www.who.int/mediacentre/factsheets/ fs311/en/

2. Debra A , Krummel. Medical nutrition therapy for cardiovascular disease. Krause, editor. Krause's Food, Nutrition and Diet Therapy. 12th ed. Philadelphia, USA: Saunders; 2008. PP 833-64.
3. Yanagisawa M, Kurihara H, Kimura S, Tomobe $Y$, Kobayashi M, Mitsui $Y$, et al. A novel potent vasoconstrictor peptide produced by vascular endothelial cells. Nature 1988; 332: 411-5.

4. Davignon J, Ganz P. Role of Endothelial Dysfunction in Atherosclerosis. Circ J 2004; 109 (3): 27-32.

5. Mather KJ, Mirzamohammadi B, Lteif A, Steinberg $\mathrm{HO}$, Baron AD. Endothelin contributes to basal vascular tone and endothelial dysfunction in human obesity and type 2 diabetes. Diabetes 2002; 51:3517-23.

6. Amirkhizi F, Siassi F, Minaie S, Djalali M, Rahimi A, Chamari M. Is obesity associated with increased plasma lipid Peroxidation and oxidative stress in women? ARYA Atheroscler 2006; 2 (4): 189-94.

7. Vincent HK,Taylor AG. Biomarkers and potential mechanisms of obesity-induced oxidant stress in humans. Int J Obes (Lond) 2006; 30: 400-18.

8. Karatas F, Karatepe M, Baysar A. Determination of free malondialdehyde in human serum by high-performance liquid chromatography. Anal Biochem 2002; 311: 76-9.

9. Adly AM . Oxidative stress and disease: An updated review. Res J Immunol 2010; 3 (2): 129-45.

10. Wei M, Gaskill SP, Haffner SM, Stern MP. Waist Circumference as the Best Predictor of Noninsulin Dependent Diabetes Mellitus (NIDDM) Compared to Body Mass Index, Waist/hip Ratio and Other Anthropometric Measurements in Mexican Americans- A 7-Year Prospective Study. Obes Res 1997; 5(1): 16-23.

11. Waist circumference and waist-hip ratio: report of a WHO expert consultation. World health organization, 2008. Geneva. (Accessed on February 8, 2014). Available from http: // www.who.org

12. Amanullah M, Zaman GS, Rahman J, Rahman SS . Lipid peroxidation and the levels of antioxidant enzymes in hypertension. Free Radicals and Antioxidants 2012; 2 (2): 12-8. 80

13. Gutteridge J, Halliwell B . The measurement and mechanism of lipid peroxidation in biological systems. Trends Biochem Sci 1990; 15: 129-35.

14. Caballero $A E$. Endothelial Dysfunction in Obesity and Insulin Resistance: A Road to Diabetes and Heart Disease. Obes Res 2003; 11 : 1278-89.

15. Maeda S, Jesmin S, lemitsu M, Otsuki T, Matsuo T, Ohkawara K. Weight Loss Reduces Plasma Endothelin-1 Concentration in Obese Men. EBM 2006; 231: 1044-7.

16. Lilyasari O, Kosasih A, Liastuti L, Librantoro, Gunawan I R, Idham I, et al. Increased Circulating Endothelin-1 in Obesity-related hypertension, but not in Obesity or Hypertension Alone. Circ J 2007; 116 (2): 341-4.

17. Cerri E. Obesity and endothelial dysfunction: mechanisms, method development and 
interventions. PhD thesis. University of Birmingham Institutional Research Archive. eTheses Repository; 2009. [Online] [Accessed on 14th, Oct, 2012]. Available from: http://etheses.bham.ac.uk/393/

18. Selvakumar C, Maheshwari $U$, Suganthi, Archana . Oxidant-Antioxidant disturbance in men classified as obese according to the preliminary WHO guidelines for Asians. JSPB 2012; 8 (1): 172-81.

19. Lachieitner $M$, Koch $T$, Harold $M$, Dzien $A$, Hoppiahler F. Tumor necrosis factor-alpha plasma level in patients with type 1 diabetes mellitus and its association with glycaemic control and cardiovascular risk factors. J Intern Med 2000; 248: 67-76.

20. El-Mesallamy $H$, Suwailem S, Hamdy $N$. Evaluation of C-Reactive Protein, Endothelin-1, Adhesion Molecule(s), and Lipids as Inflammatory Markers in Type 2 Diabetes Mellitus Patients. Mediators Inflamm; 2007.(Accessed on 28th, Aug, 2012), Available from: http://www.ncbi.nlm.nih.gov/ pmc/articles/PMC1820618/ 